
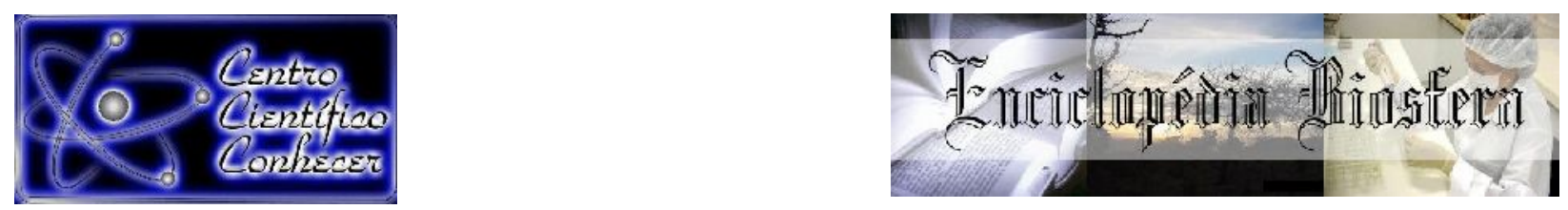

\title{
AVALIAÇÃO DE FATORES DE RISCO PARA DIABETES MELLITUS TIPO 2 ENTRE ESTUDANTES DA UNIVERSIDADE ESTADUAL DO SUDOESTE DA BAHIA- BA
}

Yane Neves Valadares ${ }^{1}$, Rafaela Brito Ribeiro dos Santos², Anny Luelly Oliveira e Oliveira $^{2}$, Erlon Oliveira Xavier², Murilo Marques Scaldaferri

${ }^{1}$ Graduanda em Ciências Biológicas pela Universidade Estadual do Sudoeste da Bahia campus Itapetinga - Brasil. Email: yane.neves16@hotmail.com

'Graduandos em Ciências Biológicas pela Universidade Estadual do Sudoeste da Bahia campus Itapetinga - Brasil

${ }^{3}$ Professor do Departamento de Ciências Exatas e Naturais da Universidade Estadual do Sudoeste da Bahia - Brasil

\section{Recebido em: 15/08/2021 - Aprovado em: 15/09/2021 - Publicado em: 30/09/2021} DOI: 10.18677/EnciBio 2021C9

\section{RESUMO}

A Diabete mellitus 2 (DM tipo 2) é a forma mais comum de Diabetes Mellitus e está relacionada à diversas complicações de saúde na população mundial. Objetivando verificar os fatores de risco para DM2 em alunos de uma instituição de ensino superior foi aplicado um questionário acerca dos hábitos alimentares e de estilo de vida dos discentes, bem como aplicação de testes recomendados pela OMS: aferição de pressão arterial, peso, IMC e relação cintura-quadril (RCQ); os dados foram posteriormente tabelados e analisados para construção do estudo. Oitenta e cinco por cento dos discentes estavam na faixa etária de 17 à 27 anos e 3\% acima dos 40 , dos quais a maior parte não prática exercícios físicos regulares (59\%) e o restante (41\%) afirmaram praticar. O IMC de $64 \%$ foi classificado como normal; $7 \%$ abaixo do peso e $29 \%$ acima. No tocante ao RCQ das mulheres, $59 \%$ estavam dentro do padrão saudável de acordo com a OMS $(<0,85 \mathrm{~cm})$ e $7 \%$ acima do ideal $(>0,85 \mathrm{~cm})$; No grupo dos homens $30 \%$ estavam no índice ideal $(<0,90 \mathrm{~cm})$ e $4 \%$ do esperado $(>0,90 \mathrm{~cm})$. A pressão arterial de $46 \%$ foi considerada baixa, $19 \%$ alta e $35 \%$ com valores considerados na faixa de normalidade. Foram identificados fatores de risco nos entrevistados, sendo o sobrepeso e o sedentarismo os mais relevantes, posto isso, a Universidade pode promover estratégias de disseminação de informações e programas de melhora da saúde dos seus estudantes.

PALAVRAS-CHAVE: Ensino Superior; Prevenção; Saúde. 


\title{
ASSESSMENT OF RISK FACTORS FOR TYPE 2 DIABETES MELLITUS AMONG STUDENTS AT THE STATE UNIVERSITY OF SOUTHWESTERN BAHIA-BA
}

\begin{abstract}
Diabetes mellitus 2 (DM tipe 2) is the most common form of Diabetes Mellitus and is related to several health complications in the world population. Aiming to verify the risk factors for DM2 in students from a higher education institution, a questionnaire was applied about the students eating habits and lifestyle, as well as the application of tests recommended by the WHO: measurement of blood pressure, weight, BMI and waist-hip ratio (WHR); the data were later tabulated and analyzed for the construction of the study. Eighty five percent of the students were aged between 17 and 27 years, and 3\% over 40, most of them did't practice regular physical exercise $(59 \%)$ and the rest (41\%) claimed to practice. The 64\% BMI was classified as normal; $7 \%$ underweight and $29 \%$ above. Regarding the women's WHR, 59\% were within the healthy standard according to the WHO $(<0.85 \mathrm{~cm})$ and $7 \%$ above the ideal $(>0.85 \mathrm{~cm})$; In the group of men, $30 \%$ were in the ideal index $(<0.90 \mathrm{~cm})$ and $4 \%$ of the expected $(>0.90 \mathrm{~cm})$. Blood pressure of $46 \%$ was considered low, $19 \%$ high and $35 \%$ with values considered within the normal range. Risk factors have been identified in the students, in which overweight and sedentary lifestyle being the most relevant, therefore, the University can promote information, dissemination of strategies and programs to promote student health.
\end{abstract}

KEYWORDS: Health; Prevention; University Education.

\section{INTRODUÇÃO}

O Diabetes mellitus (DM) pertence ao grupo de doenças que causa hiperglicemia. As manifestações da hiperglicemia são perda de peso, poliúria, polifagia, hiperfagia e visão turva. A hiperglicemia crônica pode causar vários prejuízos como dano, disfunção e falência de vários órgãos, principalmente coração, rins, nervos, vasos sanguíneos e olhos (PAIVA et al., 2001).

Uma das doenças mais comuns na infância é a DM tipo 1 na qual ocorre, geralmente, um distúrbio autoimune com produção de auto-anticorpos contra as células $\beta$ das ilhotas de Langerhans e, consequentemente, leva a queda na produção de insulina requisitando o uso de insulinoterapia para o resto da vida (FOSS et al., 2005). Esta patologia pode levar sintomas: poliúria, polidipsia, polifagia e vista embaçada. Esta enfermidade está ligada ao braço curto do cromossomo 6 do sistema HLA (Ántigeno Leucocitário Humano) (FERRARI et al., 2019; NOGUEIRA et al., 2019).

Diferente do citado acima, o diabetes mellitus tipo 2 (DM tipo 2) é um mecanismo patogênico distinto, pois a hiperglicemia crônica é causada, predominantemente, por resistência da célula alvo (muscular, adiposa e hepática) à ação da insulina circulante. Esta síndrome clínica cuja expressão fenotípica pode variar, não possui uma etiologia específica e é considerada uma doença de natureza poligênica, tendo como principal característica à disfunção bi hormonal do pâncreas. A consequente desregulação dos mecanismos de homeostasia do nível sanguíneo de glicose, ao longo do tempo, promove dano, disfunção e falha de diferentes órgãos, especialmente olhos, rins, nervos, coração e vasos sanguíneos (SANTOS et al., 2015). A DM tipo 2 é a forma mais comum de Diabetes Mellitus que se caracteriza por defeitos na secreção e atuação da insulina, os problemas relacionados a esses defeitos podem ser observados quando há manifestação de 
hiperglicemia, geralmente os pacientes que possuem o diabetes mellitus tipo 2 apresentam sobrepeso ou obesidade (SBD, 2020; NOGUEIRA et al., 2019).

A Organização Mundial da Saúde (OMS, 2009) estipula que os altos índices de glicemia são o terceiro fator relacionado à mortalidade prematura em uma escala de importância. Segundo a Federação Internacional de Diabetes (IDF, 2017) 8,8\% da população mundial com 20 a 79 anos de idade (correspondendo 424,9 milhões de pessoas) vivem com diabetes. Potencializando um problema, visto que muitos governos, sistemas de saúde pública, particulares e mesmo os profissionais da área da saúde não concebem a real relevância do diabetes e suas possíveis complicações. Esses fatores aliados ao início silencioso dos sintomas ou progressão do DM tipo 2, fazem com que essa condição possa permanecer não detectada por vários anos, gerando possíveis outras complicações patológicas que a esta estão associadas. Estima-se que cerca de metade dos casos de diabetes mellitus tipo 2 em adultos não seja devidamente diagnosticado, sendo $84,3 \%$ dos casos em países em desenvolvimento como o Brasil (SBD, 2020).

Além das consequências citadas decorrentes desta enfermidade, a pandemia da covid-19 pode trazer mais problemas aos diabéticos. Zhou e colaboradores (2021) relatam que os efeitos lesivos na imunidade do hospedeiro, pode aumentar principalmente o risco de suscetibilidade e gravidade infecciosa. Em um estudo relatado por Richardson et al. (2020) foi constatado que dos 5.700 pacientes de Nova York 33,8\% tinham diabetes, além disso estes pacientes foram predispostos a desenvolver infecções graves. Diante disso, destaca-se a importância de medidas de controle para o DM e mais pesquisas na área sobre o mesmo.

Perante o exposto é necessário evidenciar que o controle do diabetes, só pode ser alcançado ao se estabelecer e desenvolver laços de trabalho entre órgãos governamentais e sociedade civil organizada, visando a prevenção e controle do diabetes mellitus tipo 2, para tanto, pesquisas frequentes na área são sempre necessárias (PEREIRA; FIGUEIREDO, 2017). Dessa forma, o presente trabalho teve como objetivo verificar os fatores de risco para Diabetes Mellitus tipo 2 em alunos de uma Instituição de ensino superior.

\section{MATERIAL E METODOS}

Foi realizado um estudo de natureza quantitativa descritiva utilizando como instrumento de coleta de dados um questionário previamente elaborado contendo 17 questões fechadas referente aos principais fatores de risco para diabetes mellitus tipo 2 e hábitos cotidianos dos entrevistados. O estudo foi realizado na Universidade Estadual do Sudoeste da Bahia, campus Itapetinga (BA), onde foram aplicados os questionários para 100 alunos da instituição de diferentes cursos e semestres devidamente matriculados em algum curso na instituição.

Dentro do questionário foram analisadas variáveis como: gênero (masculino e feminino), presença de familiares com a doença de $1^{\circ}$ grau (pais e irmãos) e $2^{\circ}$ grau (avós, tios e primos). O índice de massa corporal (IMC), foi obtido através da relação peso/altura ${ }^{2}$. Para fazer as análises com os valores do IMC os indivíduos foram classificados em: abaixo do peso (com $17 \mathrm{~kg} / \mathrm{m}^{2}$ a $18,4 \mathrm{~kg} / \mathrm{m}^{2}$ ), peso ideal (indivíduos com $18,5 \mathrm{~kg} / \mathrm{m}^{2}$ a $24,9 \mathrm{~kg} / \mathrm{m}^{2}$ ) e sobrepeso ou possível obesidade (com $25 \mathrm{~kg} / \mathrm{m}^{2}$ ou acima) seguindo os critérios propostos pela OMS (OMS, 2000).

Outro dado coletado durante a amostragem foi da relação cintura e quadril (RCQ) medindo a proporção de cintura e quadril com a utilização de uma fita métrica $(0 \mathrm{~cm}$ a $150 \mathrm{~cm})$ com intervalos de $0,5 \mathrm{~cm}$, sendo a cintura ou circunferência abdominal definida como a menor circunferência abaixo das costelas e acima do 
umbigo, e o quadril ou circunferência gluteal como a maior circunferência na extensão posterior das nádegas. Já a aferição da pressão arterial foi realizada utilizando um bracelete medidor de pressão arterial digital automático B699. Foram considerados indivíduos contendo pressão normal quando a pressão sistólica medida e mantida em torno de $120 \mathrm{mmHg}$ ou $130 \mathrm{mmHg}$ e a pressão diastólica, entre $80 \mathrm{mmHg}$ e $90 \mathrm{mmHg}$ (seguindo recomendação da OMS). Acima destes valores foram considerados com pressão alta e valores abaixo foi considerada pressão baixa.

Para avaliar a prática de atividade física foi considerada quem pratica regularmente (considerando que o sujeito realizava três ou mais atividades físicas durante a semana) e quem não pratica (apenas uma ou nenhuma vez na semana). Outra variável analisada foi o consumo de álcool e a frequência, bem como, a influência da vida acadêmica no consumo de alimentos considerados pouco saudáveis, consumo de álcool e prática de exercícios físicos.

Após a coleta dos dados, todas as respostas foram transpostas para uma planilha no programa Microsoft Excel (2016) para tratamento, estruturação do banco de dados (calculando desvio padrão dos resultados tabulados) e formulação das tabelas e os gráficos produzidos através do software BioStat 2008 versão 5.0.1.

\section{RESULTADOS E DISCUSSÃO}

Dos 100 universitários entrevistados, 85\% estavam na faixa etária de 17 a 27 anos (Figura 1). A idade é um fator também levado em consideração, já que a incidência do diabetes (DM tipo 2) aumenta diretamente com o progredir da idade, especialmente após os 40 anos, quando geralmente o diabetes mellitus tipo 2 é diagnosticado (SBD, 2020). Sendo assim, apesar de apenas cerca de $3 \%$ dos entrevistados apresentarem mais de 40 anos, o aumento da idade foi analisado no desenvolvimento da doença associado a outros fatores.

FIGURA 1: Percentual de idade dos alunos entrevistados.

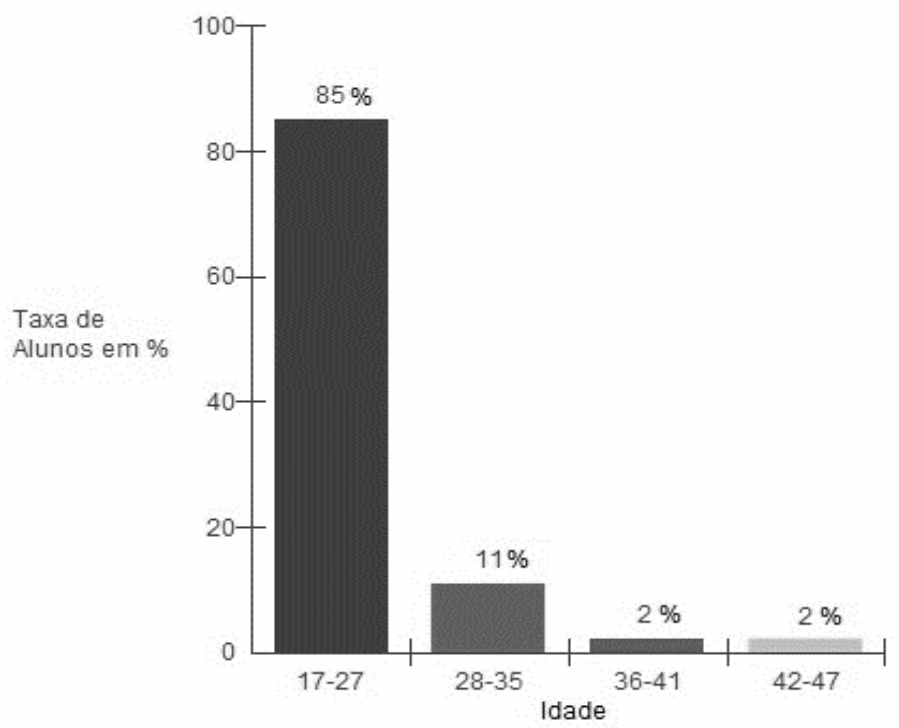

Fonte: os autores (2021)

Em relação ao gênero a maioria pertencia ao feminino (66\%) e apenas 34\% ao gênero masculino. Segundo dados da pesquisa sobre Vigilância de Fatores de Risco e Proteção para Doenças Crônicas por Inquerito Telefônico (BRASIL, 2016) a prevalência de diabetes cresceu de $5,5 \%$ para $8,9 \%$ em 2016 , tendo as mulheres maior prevalência $(9,9 \%)$. 
Outras variáveis analisadas foram em relação aos fatores de risco. O primeiro (histórico familiar) demonstrou que $66 \%$ dos entrevistados possuiam familiares já diagnosticados com diabetes e $34 \%$ não possuiam. O fator hereditário quando se trata da diabetes é um traço dominante, o que torna ainda mais fácil que o individuo herde tal característica (RACITI et al., 2015). Alguns estudos já demonstram que indivíduos com histórico familiar de diabetes mellitus tipo 2 tem até 10 vezes mais chances de desenvolver a doença quando comparados com indivíduos com iguais fatores de risco, mas sem histórico familiar (SZCZERBIŃSKI et al., 2019).

Esse valor encontrado corrobora dados relatados no estudo realizado por Pereira e colaboradores (2019) quanto aos fatores de risco não modificáveis, no qual também, mais da metade dos participantes afirmaram possuir no histórico familiar casos de diabetes (cerca de 57,8\%).

Quanto à prática de atividade física, $59 \%$ dos entrevistados afirmaram não praticar nenhum exercício e $41 \%$ afirmaram praticar. O estilo de vida sedentário é o principal responsável pela redução da queima de calorias, promovendo acúmulo de peso. Segundo Marçal e colaboradores (2018) a prática de atividade física aumenta a sensibilidade do organismo a insulina, auxiliando no controle dos níveis de glicose no sangue, diminuindo também a necessidade de hipoglicemiantes, além de auxiliar na perda de peso dos pacientes obesos, diminuindo 0 risco de doenças cardiovasculares.

Em relação ao índice de massa corporal (IMC) 64\% foram classificados como normais; $7 \%$ se encontram abaixo do peso e $29 \%$ acima do peso ideal (Figura 2). 0 sobrepeso é considerado o maior fator de risco para desenvolvimento do diabetes tipo 2, sendo que, a frequência é três vezes maior para o desenvolvimento desta doença, este dado por si só justifica a necessidade de programa de educação para esta população, através dos próprios profissionais da saúde a esta pertencente (ORTIZ; ZANETTI, 2001).

FIGURA 2: Percentual de indivíduos de acordo com sua faixa de peso.

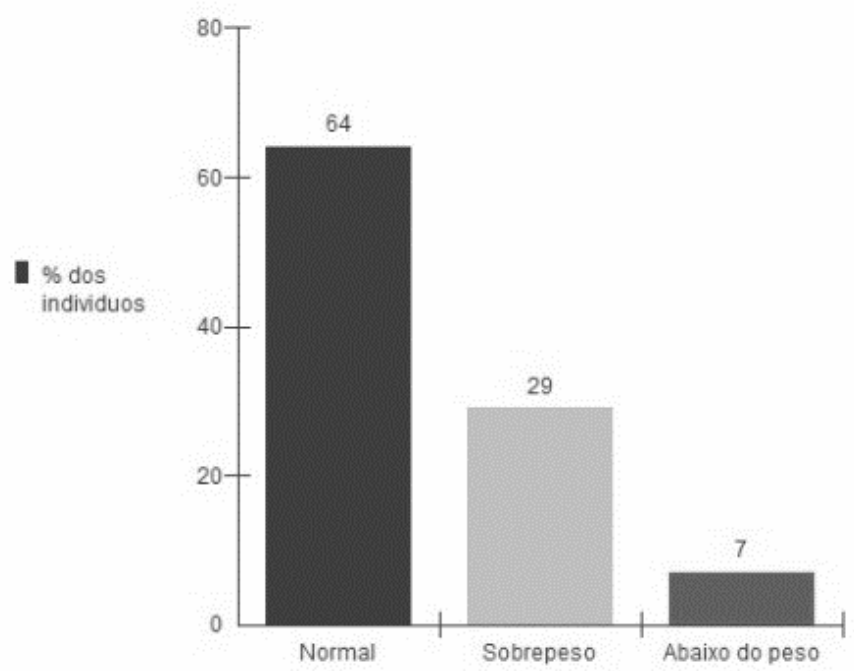

Fonte: os autores (2021)

A pesquisa realizada por Pires e Mussi (2016), com cerca de 154 graduandos do curso de enfermagem, na Universidade Federal Pública, em Salvador, Bahia, constataram que havia um terço de adultos jovens com excesso de peso, uma vez 
que $26 \%$ dos graduandos apresentaram sobrepeso e $4,5 \%$, dados que se assemelham ao encontrado na presente pesquisa.

Dentre os $29 \%$ dos indivíduos com sobrepeso entrevistados apenas $8 \%$ afirmaram não praticar nenhum tipo de atividade física e $21 \%$ afirmaram praticar algum tipo de exercício físico, dado que pode demonstrar conhecimento por parte dos entrevistados acerca da importância da prática de exercício e manutenção de um estilo de vida saudável. Estudos revelam que quanto mais inativo o indivíduo, maior o IMC. O nível de atividade física é inversamente proporcional à quantidade de gordura corporal e ao peso dos indivíduos (KOLCHRAIBER et al., 2018). Já quanto à alimentação, ainda levando em consideração o IMC dos indivíduos normais (64 no total) cerca de 34 possuíam uma alimentação de má qualidade; dos indivíduos abaixo do peso ( 7 no total) três relataram não fazer uma alimentação saudável e dos 29 indivíduos com sobrepeso 19 apresentavam uma dieta com alimentos de qualidade não recomendável (Figura 3).

FIGURA 3: Relação entre a alimentação e o IMC dos entrevistados.

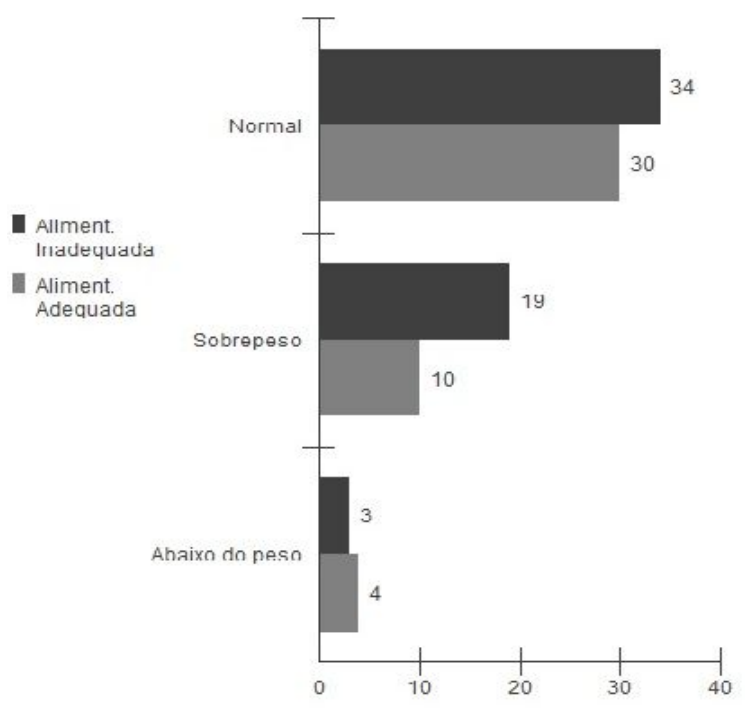

Fonte: os autores (2021)

O sobrepeso e/ou obesidade estão presentes na maioria dos pacientes com DM tipo 2. Estudos clínicos realizados demonstram que dieta com alto teor de gordura e baixo teor de fibras aumenta o risco de desenvolvimento da intolerância à glicose e do DM tipo 2, além da obesidade ser apontada como um dos principais fatores de risco para a diabetes mellitus tipo 2 . Os hábitos alimentares e a prática de atividades físicas exercem uma poderosa influência sobre o balanço energético, sendo considerados os principais fatores, passíveis de modificação, determinantes do sobrepeso e aparecimento de enfermidades como, por exemplo, o diabetes colaborando com 0 aumento da resistencia à insulina e maior risco de desenvolvimento da DM tipo 2 (ADA, 2015; MOURA et al., 2021).

Outra estimativa analisada foi da relação cintura-quadril (RCQ) na qual do total de 66 mulheres entrevistadas, $59 \%$ das mulheres estavam dentro do padrão, segundo a OMS, considerado saudável $(<0,85)$ e $7 \%$ se encontravam acima do ideal $(>0,85)$. Do total de 34 homens entrevistados $30 \%$ estavam dentro do índice considerado ideal $(<0,90)$ e $4 \%$ se encontravam acima do esperado $(>0,90)$ como indicado na figura 4. 
FIGURA 4: Relação cintura-quadril (RCQ) dos homens e mulheres entrevistados.

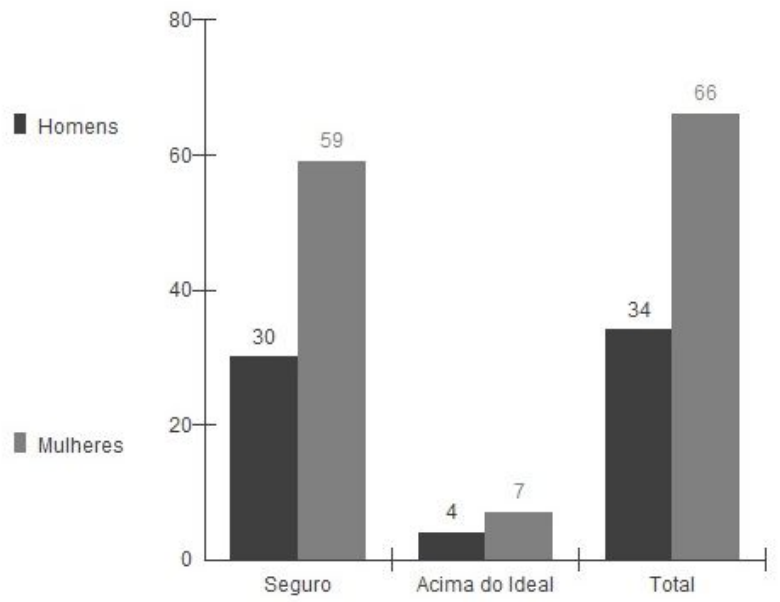

Fonte: os autores (2021).

Outro fator de risco analisado foi a pressão arterial. Dos entrevistados $46 \%$ possuíam pressão considerada baixa, 19\% apresentavam pressão alta e $35 \%$ pressão considerada normal (Figura 5). A DM tipo 2 tende a se relacionar com a hipertensão principalmente em indivíduos mais velhos, pois neste caso alguns fatores se agregam, como o envelhecimento fisiológico natural e os fatores relacionados ao estilo de vida; uma dieta desbalanceada, tabagismo, falta de exercícios físicos, bem como o histórico da família tendem a contribuir para agravamento desses quadros. Atualmente o estilo de vida é considerado um dos mais importantes pontos a se observar no desenvolvimento da DM tipo 2 (RIBEIRO et al., 2020).

FIGURA 5: Distribuição dos indivíduos quanto a pressão arterial.

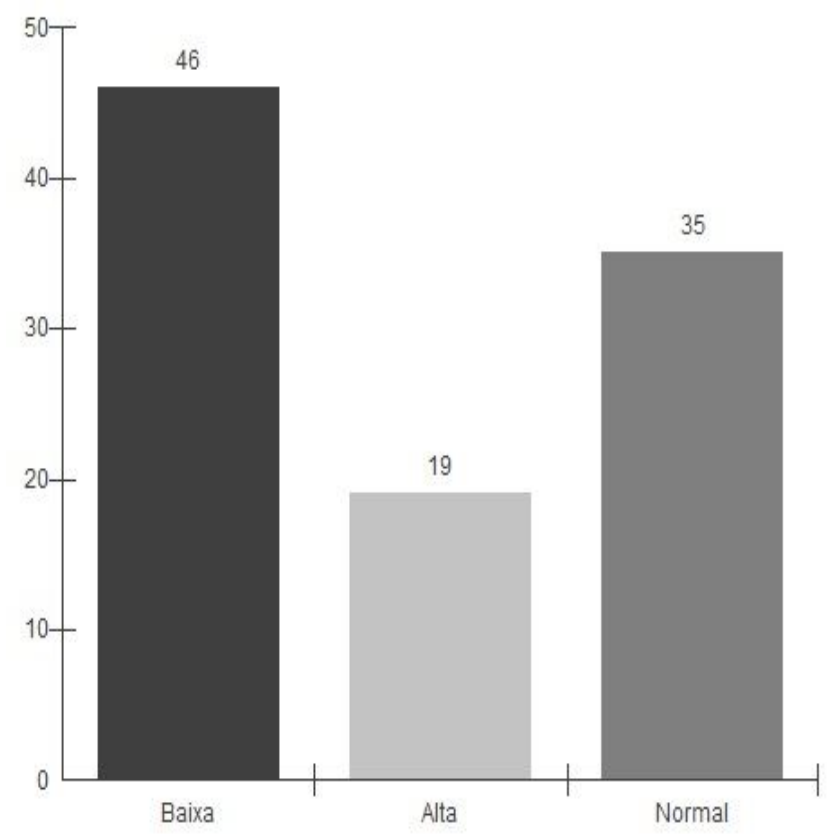

Fonte: os autores (2021) 
Dos $19 \%$ dos indivíduos que apresentavam pressão arterial alta, apenas 11 afirmaram praticar algum tipo de exercício físico. Atualmente sabe-se que a realização regular de exercícios físicos, preferencialmente os aeróbicos, promovem uma melhora nos quadros de DM tipos 1 e 2, tendo em vista que o treinamento físico gera efeitos fisiológicos benéficos e por sua vez interagem com a fisiopatologia (SANTOS et al., 2021).

Em relação ao consumo de álcool $45 \%$ afirmaram não consumir e $55 \%$ afirmaram consumir. Destes 55\%; $28 \%$ afirmaram consumir apenas uma vez na semana; $12 \%$ consomem de duas a quatro vezes; $10 \%$ consomem de cinco vezes acima na semana e apenas $5 \%$ consomem menos que uma vez na semana. Mutonni e Costa (2016) também constataram que mais de $50 \%$ dos jovens consomem bebidas alcoólicas e estudos revelam que o consumo de álcool na juventude pode se manter ao longo dos anos e este pode trazer consequências como desenvolvimento de DM tipo 2 em até 46,7\% (GOMES et al., 2012).

A últimas variáveis analisadas foram relacionando o consumo de álcool e a prática de exercícios com a rotina estudantil. Sobre a prática de atividade física $71 \%$ afirmaram que a dificuldade na prática de exercício físico está diretamente relacionada com a rotina universitária e apenas 29\% negaram (Figura 6).

Figura 6: Distribuição dos indivíduos quanto a influência da vida acadêmica na prática de exercícios.

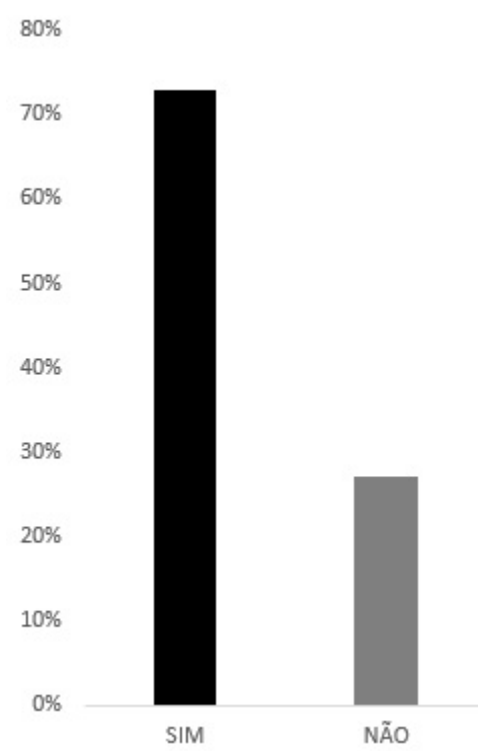

Fonte: os autores (2021)

Apesar da rotina universitária não ser considerada diretamente um fator de risco, esta pode influenciar diretamente no aumento de hábitos prejudiciais (como má alimentação e pouca prática de exercícios), como observado e que, a longo prazo, podem resultar no aparecimento de DM tipo 2 que é diretamente ligada a hábitos cotidianos (SANTOS, 2021). Por fim, a última variável relacionando a Universidade foi sobre o consumo de álcool na qual dos $55 \%$ dos indivíduos que consumiam álcool, $32 \%$ afirmaram que a rotina universitária influencia no aumento de seu consumo de álcool e $23 \%$ negaram (Figura 7 ) demonstrando como a pressão e o estresse envolvendo o ambiente universitário pode, também, levar ao consumo excessivo de álcool o que, a longo prazo, pode resultar no aparecimento da DM tipo 2 (SILVA FILHO et al., 2020; BENETON et al., 2021). 
FIGURA 7: Distribuição dos indivíduos quanto a influência da vida acadêmica com o consumo de álcool.

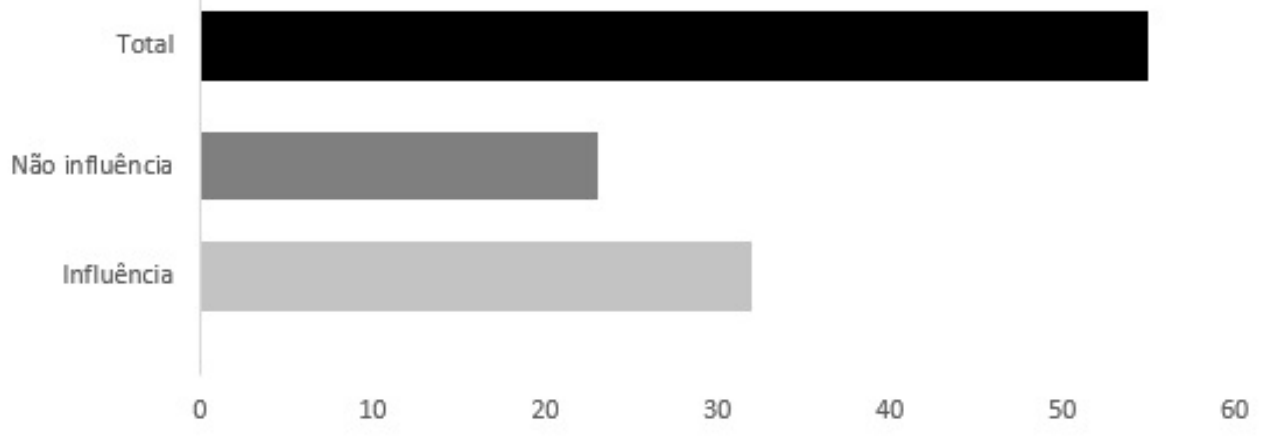

Fonte: os autores (2021).

Cardoso e colaboradores (2019) ao abordarem o estresse em universitários, sob uma visão da epidemiologia, observaram que muitos estudantes estão dispostos numa jornada extensiva, extrapolando as atividades acadêmicas, trabalho para o autossustento e obrigações domésticas e tal rotina pode gerar danos fisiológicos ao organismo desses estudantes. A associação do estresse e alcoolismo pode complicar possíveis casos de DM tipo 2, visto que são dois fatores preponderantes para o agravamento dessa condição. O estresse da vida acadêmica, caso alcance um grau mais elevado, pode fazer com 0 indivíduo desenvolva algumas caracteristicas como: irritabilidade, distúrbios de apetite, distúrbios de sono, falta de autocuidado entre outros (LEÃO et al., 2018).

Apesar de existirem poucas pesquisas relacionando rotina universitária e aparecimento de doenças como a DM tipo 2, de acordo com Rios (2006) a vida acadêmica aproxima o estudante das exigências da sociedade no que concerne a atuação profissional e cidadã, exigindo a eficácia, adaptação às novas situações e o lidar com a pressão, compromissos, responsabilidades e aceitações externas. Assim, durante o processo de formação muitos são os aspectos que comprometem a saúde integral do estudante universitário instigando hábitos incompatíveis com um estilo de vida saudável.

\section{CONCLUSÃO}

Fatores de risco para a Diabetes Mellitus como sedentarismo, predisposição genética, má alimentação e obesidade estão presentes na vida de estudantes universitários que, muitas vezes, alteram o seu estilo de vida com a entrada na vida acadêmica. A reduzida preocupação com esta patologia em jovens estudantes pode desencadear a formação de hábitos de vida não saudáveis que refletirão por toda a vida.

Assim, ao analisar todas essas variáveis entre os universitários, entendemos ser importante que as Universidades invistam em ações que orientem não só os estudantes, mas toda a população em seu entorno sobre esta grave doença que pode ser tratada e ter suas sequelas minimizadas com a adoção de hábitos saudáveis e mais qualidade de vida.

A promoção de saúde é uma importante estratégia para influenciar de forma positiva fatores determinantes para saúde. Políticas públicas com este intuito podem apresentar resultados relevantes na busca pela redução do Diabetes Mellitus no Brasil. 


\section{REFERÊNCIAS}

ADA - American Diabetes Association. Diagnosis and classification of diabetes mellitus. Diabetes Care, v. 1, n. 1, p. 8-16, 2015. Disponível em:< https://doi.org/10.2337/dc15-S005/>. DOI: 10.2337/dc15-S005

BENETON, E. R.; SCHMITT, M.; ANDRETTA, I.; Sintomas de depressão, ansiedade e estresse e uso de drogas em universitários da área da saúde. Revista da SPAGESP, v. 22, n. 1, p. 145-159, 2021. Disponivel em:< https://dialnet.unirioja.es/servlet/articulo?codigo=7816244 >.

BRASIL. Ministério da Saúde. Vigilância de Doenças e Agravos não Transmissíveis e Promoção da Saúde. Vigilância de Fatores de Risco e Proteção para Doenças Crônicas por Inquérito Telefônico (VIGITEL): estimativas sobre frequência e distribuição sociodemográfica de fatores de risco e proteção para doenças crônicas nas capitais dos 26 estados brasileiros e no Distrito Federal em 2016. Secretaria de Vigilância em Saúde, p. 160. Brasília, 2017.

Disponível em:<https://portalarquivos2.saude.gov.br/images/pdf/2018/marco/02/vigitel-brasil2016.pdf>.

CARDOSO, J. V.; GOMES, C. F. M.; PEREIRA JUNIOR, R. J.; SILVA, D. A. Estresse em estudantes universitários: uma abordagem epidemiológica. Revista de Enfermagem UFPE on-line, Recife, v. 13, n. 17, 2019. Disponível em: $<$ https://periodicos.ufpe.br/revistas/revistaenfermagem/article/view/241547>. DOI: 10.5205/1981-8963.2019.241547

FERRARI, F.; JESUS, D. S.; SALDATELLI, A.; MOTTA, M. T.; PETTO, J. Exercício físico no diabetes mellitus tipo 1: quais as evidências para uma melhor prescrição? Revista Brasileira de Fisiologia do Exercício, v. 18, n. 1, p. 38-50, 2019. Disponível em: <https://doi.org/10.33233/rbfe.v18i1.2878>. DOI: 10.33233/rbfe.v18i1.2878

FOSS, N. T.; POLON, D. P.; TAKADA, M. H.; FOSS-FREITAS, M. C.; FOSS, M. C. Dermatoses em pacientes com diabetes mellitus. Revista de Saúde Pública, v. 39, p. 677-682, 2005. Disponível em: < https://www.scielo.br/j/rsp/a/JKsVGHssxbgrkcsZmPstjJF/?format=pdf\&lang=pt>.

GOMES, E. B.; MAGALHAES, T. M.; PEREIRA, H. C. V.; SALES, I. B.; LIMA, F. E. B. et al. Fatores de risco cardiovascular em adultos jovens de um município do Nordeste brasileiro. Revista Brasileira de Enfermagem, v. 65, p. 594-600, 2012. Disponivel em: <https://doi.org/10.1590/S0034-71672012000400007>. DOI: $10.1590 /$ S0034-71672012000400007

IDF - International Diabetes Federation .; Diabetes Atlas. 8th Edition. Bruxelas, $2017 . \quad$ Disponível em:< https://diabetesatlas.org/upload/resources/previous/files/8/IDF_DA_8e-EN-final.pdf>.

KOLCHRAIBER, F. C; ROCHA, J. S.; CESAR, D. J.; MONTEIRO, O. O.; FREDERICO, G. A. et al.; Nível de atividade física em pessoas com diabetes mellitus tipo 2. Revista Cuidarte, v. 9, n. 2, p. 2105-2116, 2018. Disponível em: < 
https://www.redalyc.org/jatsRepo/3595/359557443002/359557443002.pdf>.

LEÃO, A. M.; GOMES, I. P.; FERREIRA, J. M.; CAVALCANTI, L. P. G. Prevalência e fatores associados à depressão e ansiedade entre estudantes universitários da área da saúde de um grande centro urbano do nordeste do Brasil. Revista Brasileira de Educação Médica, Brasília, DF, v. 42, n. 4, p. 55-65, 2018. Disponível em: <https://www.scielo.br/j/rbem/a/kyYq35bwkZKHpKRTjyqjMYz/?lang=pt>. $\quad$ DOI: 10.1590/1981-52712015v42n4RB20180092

MARÇAL, D. F. D. S.; ALEXANDRINO, E. G.; CORTEZ, L. E. R.; BENNEMANN, R. M. Efeitos do exercício físico sobre diabetes mellitus tipo 1: uma revisão sistemática de ensaios clínicos e randomizados. Journal of Physical Education, v. 29, 2018. Disponível em:<https://doi.org/10.4025/jphyseduc.v29i1.2917>. DOI: 10.4025/jphyseduc.v29i1.2917

MOURA, E. O. R.; RUBIM, M. A.; OLIVEIRA, D. V.; NASCIMENTO, M. A. N.; PINA, F. L. C. Estado nutricional e hábitos alimentares de universitários: estudo em município de pequena densidade populacional. Saúde e Desenvolvimento Humano, v. 9, n. 2, 2021. Disponível:<http://dx.doi.org/10.18316/sdh.v9i2.6852>. DOI:10.18316/sdh.v9i2.6852

MUTTONI, S. M. P; COSTA, O. F. S. Padrão alimentar de adolescentes portadores de Diabetes Mellitus Tipo 1, atendidos no ambulatório de um centro de referência de Porto Alegre/RS. Saúde e Desenvolvimento Humano, v. 4, n. 2, p. 51-59, 2016. Disponível em: https://revistas.unilasalle.edu.br/index.php/saude_desenvolvimento/article/viewFile/2 317-8582.16.30/pdf >.

NOGUEIRA, B. C. M.; SOUZA, C. A.; MANZANOA, R. M.; ROSA, C. S. C.; BARRILE, S. R. Aspectos emocionais e autocuidado de pacientes com Diabetes Mellitus Tipo 2 em Terapia Renal Substitutiva. Cadernos Brasileiros de Terapia Ocupacional, v. 27, p. 127-134, 2019. Disponível em: <https://doi.org/10.4322/25268910.ctoAO1575>. DOI: 10.4322/2526-8910.ctoAO1575

ORTIZ M. C. A.; ZANETTI M. L. Levantamento dos fatores de risco para diabetes mellitus tipo 2 em uma instituição de ensino superior. Rev Latino-Am Enfermagem vol. 9, n 3, p. 58-63, 2001. Disponível em:<https://doi.org/10.1590/S010411692001000300009. DOI: 10.1590/S0104-11692001000300009

PAIVA, C. Novos critérios de diagnóstico e classificação da diabetes mellitus. Medicina Interna. v. 7, n. 4, p. 234-238, $2001 . \quad$ Disponível:< https://www.spmi.pt/revista/vol07/ch6_v7n4a2000.pdf>.

PEREIRA, M. F. V.; FIGUEIREDO, A. M. A importância do disgnóstico da Diabetes Mellitus tipos 1 e 2 na infância. SALUSVITA, Bauru, v. 36, n. 2, p. 601-614, 2017. Disponível em:

https://secure.unisagrado.edu.br/static/biblioteca/salusvita/salusvita_v36_n2_2017_a rt_15.pdf $>$.

PEREIRA, V. S. S.; REIS, D. A.; DE OLIVEIRA, S. A. Fatores de risco para 
desenvolvimento de diabetes mellitus do tipo 2 em estudantes universitários do curso de enfermagem do interior do Amazonas. Revista Eletrônica Acervo Saúde, v. $11, \quad$ n. $14, \quad$ p. e1033-e1033, 2019. Disponível em:< https://doi.org/10.25248/reas.e1033.2019>. DOI: 10.25248/reas.e1033.2019

PIRES, C. G. S.; MUSSI, F. C. Excesso de peso em universitários ingressantes e concluintes de um curso de enfermagem. Escola Anna Nery, v. 20, 2016. Disponível em:<https://doi.org/10.5935/1414-8145.20160098>. DOI: 10.5935/14148145.20160098

RACITI, G. A. LONGO, M.; PARRILLO, L.; CICCARELLI, M.; MIRRA, P. et al. Understanding type 2 diabetes: from genetics to epigenetics. Acta diabetológica, $v$. 52, $\quad$ n. $\quad 5, \quad$ p. $821-827, \quad 2015 . \quad$ Disponível em: $<$ https://www.researchgate.net/profile/Luca-

Parrillo/publication/274513916_Understanding_type_2_diabetes_from_genetics_to_e pigenetics/links/55a4d83908ae5e82ab1f6267/Understanding-type-2-diabetes-fromgenetics-to-epigenetics.pdf>. DOI: 10.1007/s00592-015-0741-0.

RIBEIRO, D. R.; CALIXTO, D. M.; SILVA, L. L.; ALVES, R. P. C. N. Prevalência de Diabetes Mellitus e Hipertensão Em Idosos. Revista Artigos.Com, v. 14, p. e2132, $2020 . \quad$ Disponível https://acervomais.com.br/index.php/artigos/article/view/2132/1208>.

em:<

RICHARDSON, S.; HIRSCH, J. S.; NARASIMHAN, M.; CRAWFORD, J. M.; MCGINN, T. et al. Presenting characteristics, comorbidities, and outcomes among 5700 patients hospitalized with COVID-19 in the New York City area. Jama network, v. $323, \quad$ n. $20, \quad$ p. 2052-2059, 2020. Disponível em:<https://jamanetwork.com/journals/jama/fullarticle/2765184/>. 10.1001/jama.2020.6775.

RIOS, O. F. L. Níveis de estresse e depressão em estudantes universitários. Dissertação de mestrado. Pontifícia Universidade Católica, São Paulo. 2006.

SANTOS, C. M. M. L.; LIRA-JUNIOR, R.; FISCHER, R. G.; SANTOS, A. P. G.; OLIVEIRA, B. H. Systemic antibiotics in periodontal treatment of diabetic patients: a systematic review. PLoS One, v. 10, n. 12, p. e0145262, 2015. Disponível em: $<$ https://journals.plos.org/plosone/article?id=10.1371/journal.pone.0145262>. $\quad$ DOI: 10.1371/journal.pone.0145262.

SANTOS, G. O. SANTOS, L. L. SILVA, D. N. SILVA, S. L. Exercícios físicos e diabetes mellitus: Revisão. Brazilian Journal of Development, v. 7, n. 1, p. 88378847, 2021. Disponível em:<https://doi.org/10.34117/bjdv7n1-599>. DOI: 10.34117/bjdv7n1-599.

SBD. Sociedade Brasileira de Diabetes. Diretrizes da Sociedade Brasileira de Diabetes. São Paulo: A C Farmacêutica. 2020. Disponível em: <http://www.saude.ba.gov.br/wp-content/uploads/2020/02/Diretrizes-SociedadeBrasileira-de-Diabetes-2019-2020.pdf>.

SZCZERBIŃSKI, Ł. GOSCIK, J.; BAUER, W.; WAWRUSIEWICZ-KURYLONEK, N. 
PACZKOWSKA-ABDULSALAM, M. et al. Efficacy of family history, genetic risk score, and physical activity in assessing the prevalence of type 2 diabetes. Polish Archives of Internal Medicine, v. 129, n. 7-8, p. 442-50, 2019. Disponível em: <https://doi.org/10.20452/pamw.14866>. DOI: doi: 10.20452/pamw.14866

SILVA FILHO, B. F. ; SANTOS, R. N.; SOUZA, A. J.; CONSTANCIO, J. F.; MUNARO, H. L. R. Fatores associados ao estresse em universitários. Research, Society and Development, v. 9, n. 9, p. e480997129-e480997129, 2020. Disponível em:<https://doi.org/10.33448/rsd-v9i9.7129>. DOI: 10.33448/rsd-v9i9.7129

WHO - World Health Organization. Global health risks: mortality and burden of disease attributable to selected major risks. Genebra, 2009. Disponivel em:<https://apps.who.int/iris/handle/10665/44203>.

WHO - World Health Organization. Obesity: preventing and managing the global epidemic. 2000. Disponível em: <https://www.researchgate.net/profile/YehyaSalih/post/Article-on-overweight-obesity-and-incident-

asthma/attachment/5d84aaeb3843b0b982652459/AS\%3A805156153204747\%4015 68975594689/download/OBESITY-+PREVENTING+AND+MANAGING.pdf >.

ZHOU, Y. CHI, J.; LV, W.; WANG, Y. et al. Obesity and diabetes as high risk factors for severe coronavirus disease 2019 (Covid 19). Diabetes/Metabolism Research and Reviews, v. 37, n. 2, p. e3377, 2021. Disponível em: <https://doi.org/10.1002/dmrr.3377>. DOI: 10.1002/dmrr.3377. 